\title{
The path to metallicity: synthesis of CNO elements in standard BBN
}

\author{
F. Iocco ${ }^{1,2}$, G. Mangano ${ }^{1}$, G. Miele ${ }^{1}$, O. Pisanti ${ }^{1}$, and P. D. Serpico ${ }^{3}$ \\ ${ }^{1}$ Dipartimento di Scienze Fisiche Università di Napoli "Federico II" and INFN Sezione di Napoli, via Cintia, 80126 Naples, Italy \\ ${ }^{2}$ Kavli Institute for Particle Astrophysics and Cosmology PO Box 20450, Stanford, CA 94309, USA \\ ${ }^{3}$ Center for Particle Astrophysics, Fermi National Accelerator Laboratory, Batavia, IL 60510-0500 USA
}

(Dated: October 10, 2018)

\begin{abstract}
We perform a reanalysis of the production of CNO elements in a standard Big Bang Nucleosynthesis scenario. The CNO yields in BBN are suppressed by the low density of the plasma, Coulomb barrier effects and the short time scales involved. Yet, the inclusion of nuclides and reactions traditionally disregarded may lead to an increase relevant enough to affect the pristine Population III stars. After a critical reanalysis and upgrade of the nuclear network our results show no major discrepancies with the ones obtained using a smaller nuclear network. The robustness of the standard predictions - the early generation of star developed in a metal-free environment - is confirmed.
\end{abstract}

PACS numbers: 26.35.+c, 97.20.Wt DSF-03/2007, FERMILAB-PUB-07-024-A, SLAC-PUB-12332

\section{PRIMORDIAL NUCLEOSYNTHESIS AND POPULATION III STARS}

Standard Big Bang Nucleosynthesis (BBN) is a oneparameter theory, mostly depending on well known nuclear and particle physics processes at the $\mathrm{keV}-\mathrm{MeV}$ scale. The only free parameter is the baryon fraction $\omega_{b}$, which can be presently determined by Cosmic Microwave Background (CMB) anisotropy [1]. Using the CMB value for $\omega_{b}$ turns BBN into a parameter-free (and thus highly predictive) theory, which can be used to check the internal consistency of the standard cosmological model, to constrain astrophysical mechanisms like ${ }^{7} \mathrm{Li}$ and ${ }^{3} \mathrm{He}$ stellar reprocessing [2, 3], to probe the cosmic neutrino background and/or constrain exotic physics, as for example in $[4,5,6,6,7,8,9,10]$.

In recent analyses like [11, 12, 13] the nuclear channels for the synthesis of light elements up to mass number $A=7$ have been carefully studied. The relevant reaction rates have also been updated with the most recent experimental and theoretical estimates, thus reducing the uncertainties on the light element abundances. Indeed, a major uncertainty on nuclear abundances arises from the uncertainty on nuclear rates. However, a necessary pre-requisite is that all the reactions relevant for the synthesis of the elements of interest are included. The widely used Wagoner-Kawano code [14] contains nuclides up to ${ }^{16} \mathrm{O}$, but no detailed analysis of the completeness of the network for mass number $A>7$ is present in the literature. Indeed, it is well known that the low density of the plasma, the higher Coulomb barrier, and the short timescales involved in BBN conspire to produce only small amounts of these elements. Yet, although being of negligible entity for the "traditional" BBN predictions of light element yields, missing reactions might have huge effects on the synthesis of "metals" - elements with mass number $A \geq 12$, whose sum we shall denote in the following with $\mathrm{M}$-in particular of the isotopes of Carbon, Nitrogen and Oxygen (CNO elements). The astrophysical properties of stars formed from the collapse of the very first structures in the Universe ("PopIII stars") may depend on the chemical composition of the cloud [15, 16]. For example, metallicities as low as $10^{-5 \pm 1}$ of the solar one (i.e. number fraction of metals as low as $\mathrm{M} / \mathrm{H} \simeq 10^{-8 \pm 1}$ ) might affect the formation of the first generation of low-mass stars [17]. These considerations motivated the present study, whose purpose is to explore the CNO synthesis channels in BBN and put sound constraints on their abundances by checking the consistency of the nuclear network. We anticipate that our results essentially confirm the robustness of the standard prediction that the early generation of star developed in a metal-free environment.

This paper is structured as follows: in Sec. [1we review the nucleosynthesis path leading to CNO elements in the standard BBN code, while in Sec. [II] we describe the way this standard nuclear network has been updated and the results we obtain. Finally, in Sec. IV we draw our conclusions.

\section{CURRENT NUCLEAR NETWORK ANALYSIS}

We can summarize the main steps of our analysis as follows:

i) identification of relevant channels for CNO synthesis in the existing code;

ii) update of the relevant reaction rates which are already present;

iii) addition of 4 new nuclides: ${ }^{9} \mathrm{Li},{ }^{10} \mathrm{Be},{ }^{9} \mathrm{C},{ }^{10} \mathrm{C}$;

iv) addition of more than 100 nuclear reactions previously neglected.

Here we describe the points i), ii), leaving the discussion of the following ones to the next section. The CNO synthesis channels in the BBN network described in [13], (hereafter "C1") are basically the same as in the original Wagoner-Kawano code (hereafter "C0"). One simplification with respect to former analyses is that now one can fix the baryon fraction from CMB measurements, $\omega_{b}=0.0223_{-0.0009}^{+0.0007}[1]$, so that within current uncertainties, the nucleosynthetic path is uniquely determined. 
The eventual small amount of Carbon is synthesized through the direct reaction channel

$$
{ }^{7} \operatorname{Li}(\alpha, \gamma){ }^{11} \mathrm{~B}(p, \gamma){ }^{12} \mathrm{C},
$$

or the secondary one

$$
{ }^{7} \operatorname{Li}(\alpha, \gamma){ }^{11} \mathrm{~B}(d, n){ }^{12} \mathrm{C} .
$$

None of these reactions is responsible for the final abundance of ${ }^{11} \mathrm{~B}$. In fact, during $\mathrm{BBN}$ both these processes compete with the stronger ${ }^{11} \mathrm{~B}(p, \alpha) 2{ }^{4} \mathrm{He}$, which depletes all ${ }^{11} \mathrm{~B}$. The final abundance of this element is therefore provided by the $\beta$ decay of ${ }^{11} \mathrm{C}$ produced by $\alpha$ capture on ${ }^{7} \mathrm{Be}$,

$$
{ }^{7} \mathrm{Be}(\alpha, \gamma){ }^{11} \mathrm{C} \rightarrow{ }^{11} \mathrm{~B},
$$

which is Coulomb-barrier suppressed with respect to the analogous $\alpha$ capture on ${ }^{7} \mathrm{Li}$.

In principle, another possible path is via the unstable nuclei with $A=8$,

$$
\begin{aligned}
& { }^{8} \mathrm{Li}(\alpha, \gamma){ }^{12} \mathrm{~B} \rightarrow{ }^{12} \mathrm{C}, \\
& { }^{8} \mathrm{~B}(\alpha, \gamma){ }^{12} \mathrm{~N} \rightarrow{ }^{12} \mathrm{C},
\end{aligned}
$$

where ${ }^{8} \mathrm{Li}$ is produced e.g. by ${ }^{7} \mathrm{Li}(n, \gamma)$ and ${ }^{6} \mathrm{Li}(t, p)$, and ${ }^{8} \mathrm{~B}$ via ${ }^{7} \mathrm{Be}(p, \gamma)$. According to our analysis, however, these channels only provide a sub-leading contribution to the $\mathrm{CNO}$ abundances.

The production of heavier isotopes of Carbon proceeds typically by radiative proton capture on carbon nuclei, followed by a $(n, p)$ reaction. For example, ${ }^{13} \mathrm{C}$ is produced from light elements through the following path

$$
{ }^{7} \mathrm{Li}(\alpha, \gamma){ }^{11} \mathrm{~B}(p, \gamma){ }^{12} \mathrm{C}(p, \gamma){ }^{13} \mathrm{~N}(n, p){ }^{13} \mathrm{C}
$$

and ${ }^{14} \mathrm{C}$ proceeds along from ${ }^{13} \mathrm{C}$ according to

$$
{ }^{13} \mathrm{C}(p, \gamma){ }^{14} \mathrm{~N}(n, p){ }^{14} \mathrm{C} \text { or }{ }^{13} \mathrm{C}(n, \gamma){ }^{14} \mathrm{C} .
$$

Nitrogen is produced by means of proton radiative capture on Carbon nuclei - e.g. ${ }^{12} \mathrm{C}(p, \gamma){ }^{13} \mathrm{~N}$ - and Oxygen isotopes are produced with the same mechanisms illustrated so far. Note that once metals $(A \geq 12)$ have been synthesized, being thermodynamically more stable (higher binding energy per nucleon) they are not disrupted back into lighter elements. This allows one to use CNO elements as estimators of the whole metallicity $\mathrm{M}$, independently of the incompleteness of the network for $A>12$. It is also worth noticing that the mechanism for ${ }^{12} \mathrm{C}$ production in BBN is completely different from the well known 3- $\alpha$ process taking place in stars, as it proceeds through the formation of the intermediate fragile nuclide ${ }^{11} \mathrm{~B}$. This is easily explained in terms of (i) the very low density of the plasma during BBN (less than $10^{20}$ baryons $/ \mathrm{cm}^{3}$ at $T \lesssim 100 \mathrm{keV}$ ); (ii) the Coulomb suppression relevant for heavier nuclei; (iii) the short time-scales involved, of the order of minutes.
Our analysis confirms that the synthesis of CNO elements in BBN proceeds along a path involving intermediate mass nuclides $(4<A<12)$; it is therefore conceivable that by neglecting intermediate mass elements or "unusual" reactions -for stellar astrophysics standardsrelevant channels may have been omitted. This possibility is scrutinized in the following.

\section{ENLARGING THE NUCLEAR NETWORK}

The next steps of our analysis have been to modify the code $\mathrm{C} 1$ adding 4 nuclides and more than 100 nuclear reactions previously neglected. The criterion adopted for adding new nuclides is based on their half-lives $t_{1 / 2}$. We find that 4 nuclides previously neglected in the C0 code have a value of $t_{1 / 2}$ which is comparable or longer than the typical times involved in BBN. We have therefore included ${ }^{9} \mathrm{Li}\left(t_{1 / 2}=0.178 \mathrm{~s}\right),{ }^{10} \mathrm{Be}\left(t_{1 / 2}=1.5 \times 10^{6}\right.$ years $)$, ${ }^{9} \mathrm{C}\left(t_{1 / 2}=0.125 \mathrm{~s}\right),{ }^{10} \mathrm{C}\left(t_{1 / 2}=19.29 \mathrm{~s}\right)$. By adding the main reactions involving these nuclides we are including all viable intermediate channels connecting lighter to heavier elements. The reactions included for these nuclides, as well as those we added for the nuclides already included in the standard $\mathrm{C} 1$ version, have been selected on the basis of physical arguments, first of all Coulomb barrier considerations. Reactions with $Z_{1} Z_{2} \geq 12$ have not been considered as they are mostly suppressed in the late times of BBN, when metals are mainly formed, and energy is very low, namely few keV. Also, no projectiles heavier than ${ }^{4} \mathrm{He}$ have been considered, since Lithium and heavier element abundances are extremely small. For each nuclide a full network including radiative capture, stripping/pick-up and charge-exchange reactions has been implemented. Many of these reactions were missing in the original nuclear network of the $\mathrm{C} 0$. In fact, they do not have appreciable effects on the light nuclide abundances which was the principal goal of the original codes. To evaluate the missing reaction rates, extensive use has been made of compilations and on-line libraries like [18, 19, 20]. The nuclear rates for many of these reactions, when measurements or theoretical models were missing, have been estimated with simple nuclear models, of the kind presented in [21, 22]. An order of magnitude uncertainty has been assumed, according to Wagoner's prescription. We shall refer to the code including the new network and the new nuclides as "C3" code (30 nuclides and 262 reactions), while the code with an enlarged network but not including the four abovementioned nuclides is named "C2" (26 nuclides and 240 reactions). The $\mathrm{C} 2,3$ codes show only few additional subleading synthesis channels for CNO elements with respect to the $\mathrm{C} 0,1$ codes, in particular a minor difference in ${ }^{12} \mathrm{C}$ (due to the additional subleading channel $\left.{ }^{11} \mathrm{~B}(d, p){ }^{12} \mathrm{~B} \rightarrow{ }^{12} \mathrm{C}\right)$, and a slightly larger amount of ${ }^{13} \mathrm{C},{ }^{14} \mathrm{C}$ and ${ }^{14} \mathrm{~N}$, see Table I. The increase of ${ }^{13} \mathrm{C}$ and ${ }^{14} \mathrm{C}$ is due to a more efficient burning of ${ }^{12} \mathrm{C}$ through the new channels ${ }^{12} \mathrm{C}(d, p){ }^{13} \mathrm{C}$ and ${ }^{13} \mathrm{C}(d, p){ }^{14} \mathrm{C}$. Due to the 
increasing of ${ }^{13} \mathrm{C},{ }^{14} \mathrm{~N}$ abundance is also increased by the (already present) reaction ${ }^{13} \mathrm{C}(p, \gamma){ }^{14} \mathrm{~N}$.

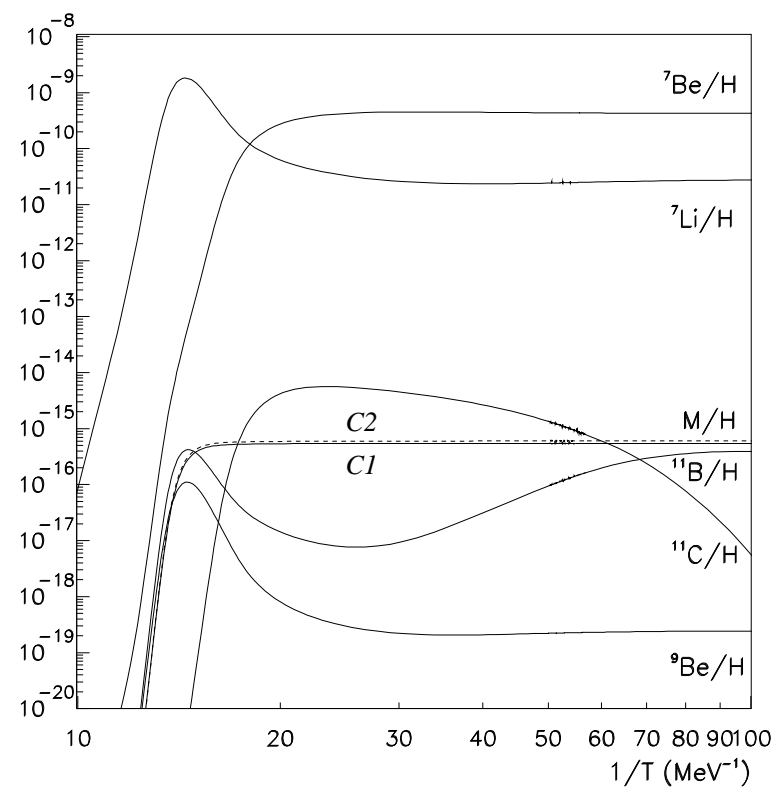

FIG. 1: The metal abundance $\mathrm{M} / \mathrm{H}$ in the $\mathrm{C} 1$ (solid line) and C2 (dashed line) codes, compared with the abundances of a few lighter elements.

On the other hand we find that the C2 and C3 codes show no appreciable difference in the final abundances, thus proving that the 4 added nuclides play no role in CNO synthesis, as reported in Table [. There are two clear conclusions that one can draw from our results: (i) an accurate (i.e. at the $10 \%$ level) prediction of the metal yield in standard BBN does depend on the details of the intermediate mass nuclide reaction network; (ii) no major change is found when enlarging the network. The physical reason is that at the beginning of the $\mathrm{BBN}$, when the higher temperature and density would favor the formation of CNO elements, their synthesis is suppressed in a high entropy Universe and by the lack of necessary intermediate elements. On the other hand, when enough "catalyzers" are produced at later times both temperature and density are not high enough to overcome the high Coulomb barrier. The evolution of metals and a few lighter elements vs. temperature is shown in Fig. 1. Note the correlation of the ${ }^{9} \mathrm{Be},{ }^{11} \mathrm{~B}$, and metal yields with the peak in the ${ }^{7} \mathrm{Li}$ production. Also note that the metal yields have a monotone behavior, due to their higher stability with respect to lighter elements.

Even taking into account a much enlarged network, one might still wonder how robust are the previous conclusions, especially given the lack of data or detailed theoretical models for several reactions. A detailed discussion of uncertainties for the order 100 new reactions is clearly unpractical. To obtain a generous but more robust upper limit to the yield of metals in standard BBN, we have performed an analysis of their yields assuming that all the reactions producing at some stage an element with $A>7$ "instantaneously" produce ${ }^{12} \mathrm{C}$, instead. In other words, a strong upper bound is obtained by assuming that all yields for nuclides with $A>7$ contribute to CNO eventually. In this way, we neglect any destruction mechanism of intermediate nuclei, the multistep nature of the synthesis of CNO nuclides, and we are maximizing the time available for their synthesis. As a result, we get $\mathrm{M} / \mathrm{H} \lesssim 10^{-10}$. This bound is clearly very conservative (about a factor $10^{5}$ higher than our best estimate), especially considering the fact that nuclides with $7<A<12$ are very fragile, and the leading reactions they are involved in typically tend to destroy them rather than producing CNO elements. It is also quite robust, as it basically depends upon the thermodynamical properties of the plasma holding for standard BBN and on the wellknown nuclear rates involving target nuclei with $A \leq 7$ only.

\begin{tabular}{|c||c|c|c|c|c|c|c|}
\hline Nuclide & $\begin{array}{c}{ }^{9} \mathrm{Be} / \mathrm{H} \\
\left(\times 10^{-19}\right)\end{array}$ & $\begin{array}{c}{ }^{11} \mathrm{~B} / \mathrm{H} \\
\left(\times 10^{-16}\right)\end{array}$ & $\begin{array}{c}{ }^{12} \mathrm{C} / \mathrm{H} \\
\left(\times 10^{-16}\right)\end{array}$ & $\begin{array}{c}{ }^{13} \mathrm{C} / \mathrm{H} \\
\left(\times 10^{-17}\right)\end{array}$ & $\begin{array}{c}{ }^{14} \mathrm{C} / \mathrm{H} \\
\left(\times 10^{-17}\right)\end{array}$ & $\begin{array}{c}{ }^{14} \mathrm{~N} / \mathrm{H} \\
\left(\times 10^{-17}\right)\end{array}$ & $\begin{array}{c}{ }^{16} \mathrm{O} / \mathrm{H} \\
\left(\times 10^{-20}\right)\end{array}$ \\
\hline \hline $\mathrm{C} 1$ & 2.4 & 3.9 & 4.4 & 7.6 & 0.6 & 2.6 & 1.8 \\
\hline $\mathrm{C} 2$ & 2.5 & 3.9 & 4.6 & 9.0 & 1.3 & 3.7 & 2.7 \\
\hline $\mathrm{C} 3$ & 2.5 & 3.9 & 4.6 & 9.0 & 1.3 & 3.7 & 2.7 \\
\hline
\end{tabular}

TABLE I: Some "heavier" nuclei yields (for $\omega_{b}=0.0223$ ) predicted by the C1 code and by using the upper limits to the production rates in the $\mathrm{C} 2$ and $\mathrm{C} 3$ codes, described in the text.

\section{CONCLUSIONS}

Besides predicting detailed values for the abundances of the light nuclei ${ }^{2} \mathrm{H},{ }^{3} \mathrm{He},{ }^{4} \mathrm{He}$, and ${ }^{7} \mathrm{Li}$, standard $\mathrm{BBN}$ also predicts that the first collapsed objects in the Universe should form in a metal free gas. In fact, Carbon 
is produced along inefficient paths involving intermediate mass elements, in particular ${ }^{11} \mathrm{~B}$ rather than the usual $3 \alpha$ reaction in stars. Very small traces of Nitrogen and Oxygen are then produced by radiative capture upon ${ }^{12} \mathrm{C}$. These predictions are relevant for determining the physical mechanisms regulating the collapse of the clouds leading to the PopIII stars, and the evolution of the smallest among these pristine stars.

Given the relevance of this topic, and the incompleteness of standard BBN nuclear networks in the mass range $A>7$, we have performed a detailed study of the synthesis channels of Carbon, Nitrogen and Oxygen in Primordial Nucleosynthesis. We have added to the standard code 4 nuclides and more than 100 reactions observing no sensible increase of CNO elements. Re-analysing the synthesis of CNO after the addition of the new reactions we find that the main channels for their production are basically the same as before and none of the newly added reactions/nuclides opens effective channels from light to heavy elements in $\mathrm{BBN}$. We consider this as a robust check that only negligible traces of metals are produced in standard BBN, in agreement with earlier and less accurate analyses. This should be regarded as a further observational test for standard $\mathrm{BBN}$, since alternative theories like Inhomogeneous BBN might lead to primordial metallicities even larger than $10^{-6}$ of the solar value (see e.g. [23, 24]).

\section{Acknowledgments}

We thank T. Abel and R. Wagoner for useful discussions. In Naples, this work was supported in part by PRIN04 of the Italian MIUR under grant Fisica Astroparticellare. PDS acknowledges support by the US Department of Energy and by NASA grant NAG 5-10842 at Fermilab.
[1] D. N. Spergel et al., "Wilkinson Microwave Anisotropy Probe (WMAP) three year results: Implications for cosmology," astro-ph/0603449

[2] P. P. Eggleton, D. S. P. Dearborn and J. C. Lattanzio, "Deep Mixing of He-3: Reconciling Big Bang and Stellar Nucleosynthesis," Science, 26 October 2006 astro-ph/0611039.

[3] A. J. Korn et al., "A probable stellar solution to the cosmological lithium discrepancy," Nature 442, 657 (2006) astro-ph/0608201.

[4] V. Barger, J. P. Kneller, P. Langacker, D. Marfatia and G. Steigman, "Hiding relativistic degrees of freedom in the early universe," Phys. Lett. B 569, 123 (2003) hep-ph/0306061.

[5] A. Cuoco et al. "Present status of primordial nucleosynthesis after WMAP: results from a new BBN code," Int. J. Mod. Phys. A 19, 4431 (2004) astro-ph/0307213.

[6] K. Jedamzik, "Did something decay, evaporate, or annihilate during big bang nucleosynthesis?," Phys. Rev. D 70, 063524 (2004) astro-ph/0402344.

[7] R. H. Cyburt, B. D. Fields, K. A. Olive and E. Skillman, "New BBN limits on physics beyond the standard model from He-4," Astropart. Phys. 23, 313 (2005) astro-ph/0408033.

[8] P. D. Serpico and G. G. Raffelt, "Lepton asymmetry and primordial nucleosynthesis in the era of precision cosmology," Phys. Rev. D 71, 127301 (2005) astro-ph/0506162.

[9] G. Mangano et al. "Relic neutrino decoupling including flavour oscillations," Nucl. Phys. B 729, 221 (2005) hep-ph/0506164.

[10] G. Mangano et al. "Effects of non-standard neutrino electron interactions on relic neutrino decoupling," Nucl. Phys. B 756, 100 (2006) hep-ph/0607267.

[11] R. H. Cyburt, "Primordial Nucleosynthesis for the New Cosmology: Determining Uncertainties and Examining Concordance," Phys. Rev. D 70, 023505 (2004) astro-ph/0401091.

[12] P. Descouvemont, A. Adahchour, C. Angulo, A. Coc and
E. Vangioni-Flam, "Compilation and R-matrix analysis of big bang nuclear reaction rates," astro-ph/0407101

[13] P. D. Serpico et al. "Nuclear Reaction Network for Primordial Nucleosynthesis: a detailed analysis of rates, uncertainties and light nuclei yields," JCAP 0412, 010 (2004) astro-ph/0408076.

[14] L Kawano, Let's go Early Universe Preprint FermilabPub-92/04-A.

[15] V. Bromm, A. Ferrara, P. S. Coppi and R. B. Larson, "The Fragmentation of Pre-enriched Primordial Objects," Mon. Not. Roy. Astron. Soc. 328, 958 (2001) astro-ph/0104271.

[16] A. K. Jappsen, S. C. O. Glover, R. S. Klessen and M. M. Mac Low, "Star formation at very low metallicity. II: On the insignificance of metal-line cooling during the early stages of gravitational collapse," Astrophys. J., in press astro-ph/0511400.

[17] R. Schneider, A. Ferrara, R. Salvaterra, K. Omukai and V. Bromm, "Low-Mass Relics of Early Star Formation," Nature 422, 869 (2003) astro-ph/0304254.

[18] Audi et al., "The Nubase evaluation of nuclear and decay properties," Nuc. Phys.A 624 (1997), 1.

[19] Table of Isotopes, 8th edition, online at http://www . tunl.duke. edu/nucldata .

[20] http://www.nndc.bnl.gov/exfor3/endf00.htm.

[21] W.A. Fowler, F. Hoyle, "Neutrino Processes and Pair Formation in Massive Stars and Supernovae," ApJ. Suppl. 9, (1964), 201.

[22] R. Wagoner, W. Fowler, F. Hoyle, "On the Synthesis of Elements at Very High Temperatures," ApJ 148 (1967), $3 \mathrm{~W}$.

[23] K. Jedamzik, G. M. Fuller, G. J. Mathews and T. Kajino, "Enhanced Heavy Element Formation In Baryon Inhomogeneous Big Bang Models," Astrophys. J. 422, 423 (1994) astro-ph/9312066.

[24] K. Jedamzik, "Did the Universe start at Zero Metallicity?," astro-ph/9911242. 